\title{
Knee Joint Osteochondral Reconstruction Using Fresh Femoral Head Autograft
}

\author{
Patrick Weinrauch ${ }^{\mathrm{a}, \mathrm{b}, \mathrm{d}}$, Richard Steer ${ }^{\mathrm{c}}$, Sharon Kermeci ${ }^{\mathrm{a}}$, Kevin Tetsworth ${ }^{\mathrm{c}}$
}

\begin{abstract}
We present the management of polyarticular osteonecrosis in a 17-year-old female, with marked symptoms involving both hips and one knee. As a complication of chemotherapy to treat leukaemia, the articular surfaces of both femoral heads and the right knee lateral femoral condyle collapsed, resulting in significant pain and disability. For this unusual case a novel technique was employed, using a method we believe has not been described previously. The articular surface of the knee was reconstructed using fresh bulk osteochondral autograft harvested from the ipsilateral femoral head during a simultaneous total hip arthroplasty. Reconstructive options in young patients with multiple joints involved are often restricted, and suitable osteochondral allografts can be difficult to obtain. Fresh osteochondral auto-grafting enabled rapid osseous integration and articular surface reconstruction using living host cartilage, resulting in an excellent clinical outcome. Although uncommon, concurrent avascular necrosis of multiple large joints is occasionally encountered, and management options are particularly limited in young patients. We recommend this innovative technique be considered in those patients who satisfy the criteria for bulk osteochondral grafting procedures where total hip replacement is also required.
\end{abstract}

Keywords: Osteonecrosis; Auto-graft; Osteo-chondral; Knee; Hip

\section{Introduction}

A 14-year-old female patient presented for evaluation of bi-

\footnotetext{
Manuscript accepted for publication October 2, 2013

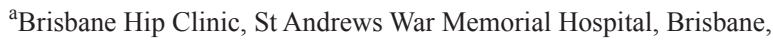
Australia

${ }^{\mathrm{b}}$ Griffith University, School of Medicine, Brisbane, Australia

'Department of Orthopaedic Surgery, Royal Brisbane and Women's Hospital, Brisbane, Australia

${ }^{\mathrm{d}}$ Corresponding author: Patrick Weinrauch, Brisbane Hip Clinic, St Andrews War Memorial Hospital, Spring Hill Q 4000, Australia.

Email: pweinrauch@brisbanehipclinic.com.au
}

doi: http://dx.doi.org/10.4021/jmc1524w lateral hip osteonecrosis she developed as a complication of chemotherapy used in the management of acute lymphocytic leukaemia. The patient reported multiple other complications due to chemotherapy including bilateral humeral head osteonecrosis, bilateral distal femoral osteonecrosis, peripheral sensory and motor neuropathy, and a bowel perforation requiring partial resection and ileostomy. The patient was intermittently wheelchair bound on the basis of her hip pathology, superimposed upon the ataxia secondary to her peripheral motor neuropathy. Over the preceding months both hips had become persistently symptomatic with moderate pain on a daily basis, with a corresponding significant reduction in function and difficulty with ambulation.

On examination the patient demonstrated an ataxic gait with hyper tonicity of the lower limb musculature and a foot drop. X-ray and MRI evaluation demonstrated significant avascular segments of both femoral heads with articular collapse (Steinberg stage 5C right side, Steinberg 4C left) (Fig. 1). Avascular necrosis of the left acetabulum was also noted. Surgical intervention for the hips was delayed due to the high risk of infection related to her ongoing chemotherapy and the presence of an ileostomy. Non-operative therapy includ-

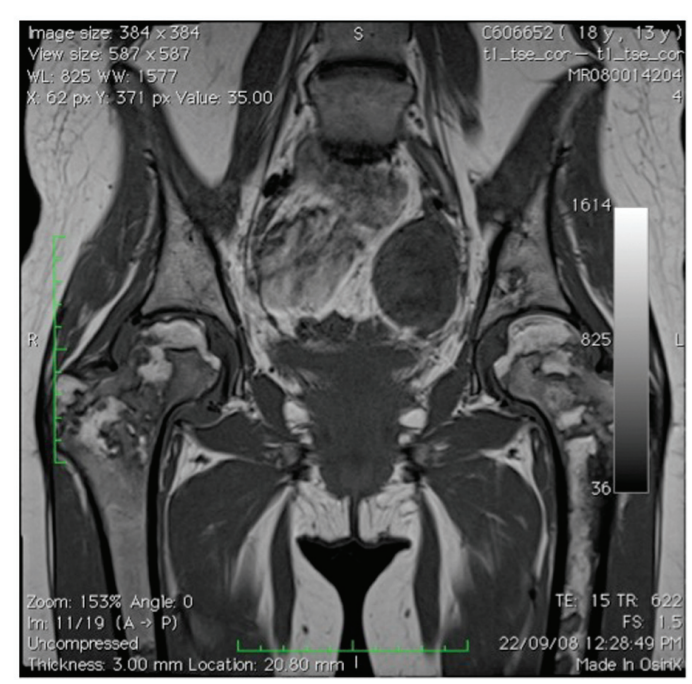

Figure 1. MRI of pelvis showing avascular segments of femoral heads (2008). 


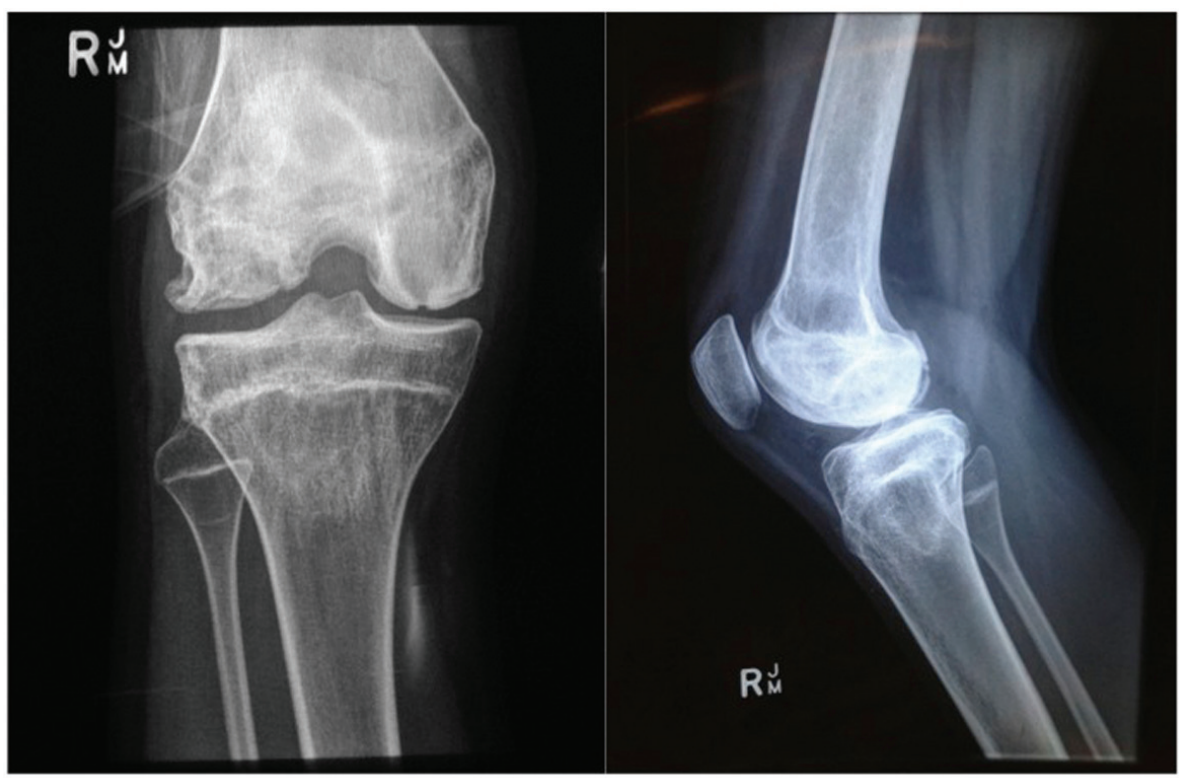

Figure 2. X-ray (weight-bearing) of AP and Lateral right knee (May 2010).

ed intravenous bisphosphonate infusions and symptomatic measures for analgesia.

Upon review at 15.5 years of age, progressive collapse of both femoral heads was noted on serial radiographs. The patient at this time also reported increasing pain about the lateral aspect of her right knee with radiographic evidence of osteonecrosis of the lateral femoral condyle (Fig. 2, 3). Drill decompression of the lateral condyle segment was conducted; however, the right knee continued to deteriorate with increasing pain, progressive stiffness, instability, and mechanical symptoms. A knee X-ray five months after the decompression procedure demonstrated progressive collapse of the right lateral femoral condyle (Fig. 4). Subsequent arthroscopy confirmed significant disruption of the articular surface of the lateral femoral condyle, with the remaining articular surfaces remaining relatively well preserved. Arthroscopic debridement with removal of loose bodies was conducted as an interim measure. The right knee joint, however, remained persistently symptomatic and treatment by bulk osteochondral allograft was recommended.

The patient, by now 17 years of age, also described increasing symptoms attributable to both hip joints. Chemotherapy had been ceased 16 months prior and no recurrence of leukaemia was detected on bone marrow aspirates. The

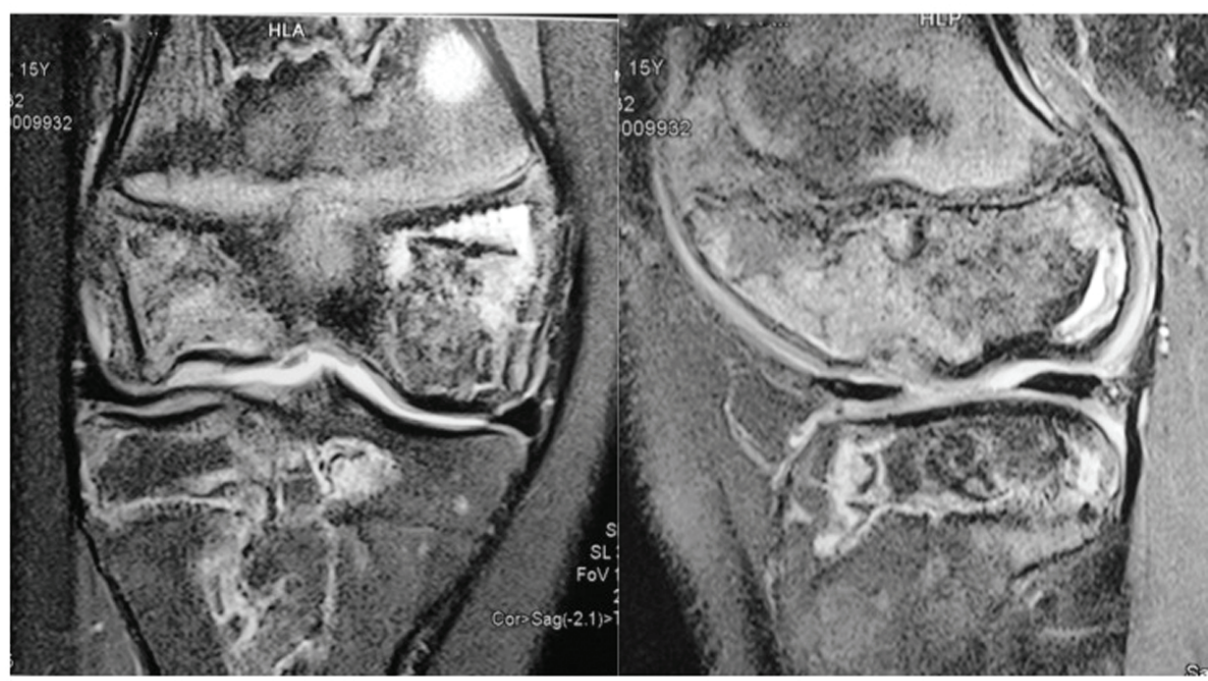

Figure 3. MRI of right knee showing areas of osteonecrosis (May 2010). 


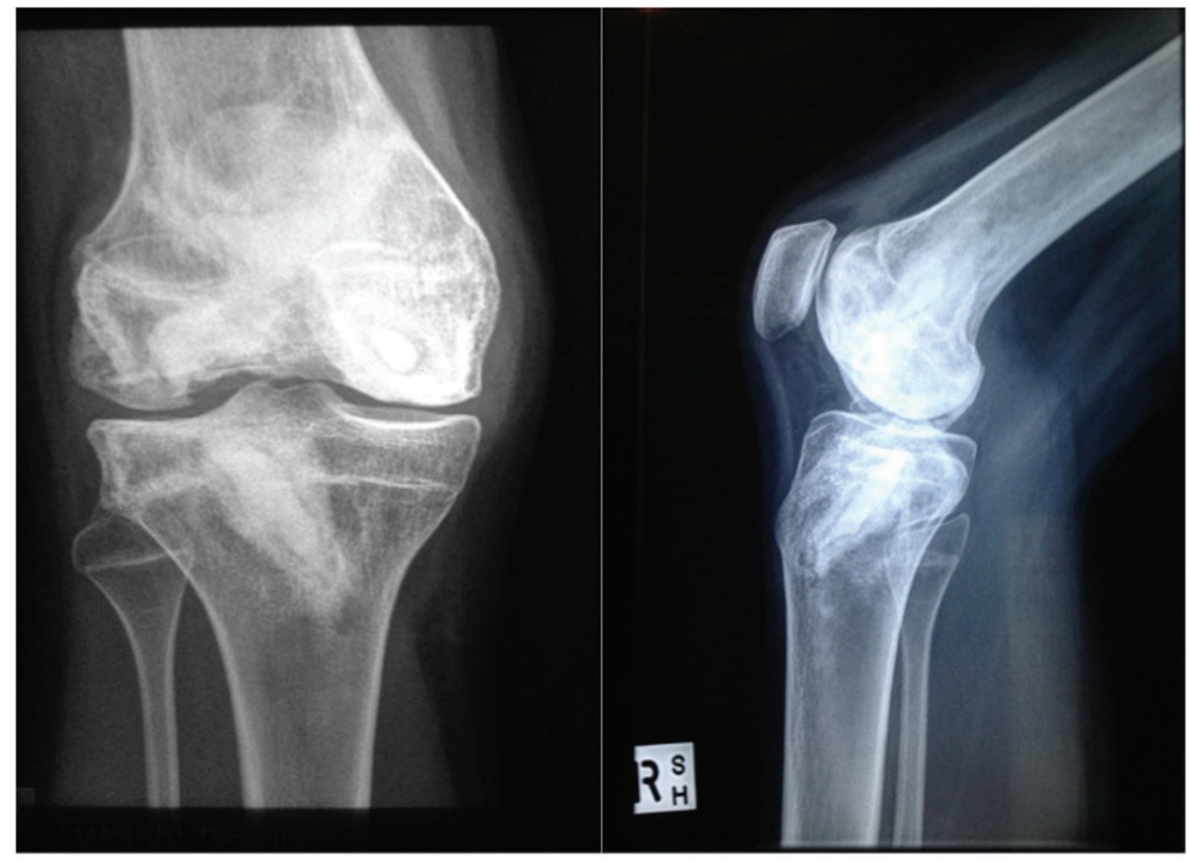

Figure 4. X-ray AP and Lateral of right knee post drilling procedure (Oct 2010).

ileostomy had been reversed and the peripheral neuropathy almost completely resolved. Considering the marked pain and significant disability resulting from the osteonecrosis of both femoral heads, bilateral total hip arthroplasty was also recommended.

\section{Surgical Technique}

Preoperative imaging demonstrated the posterior and inferior aspect of the right femoral head was relatively unaffect-

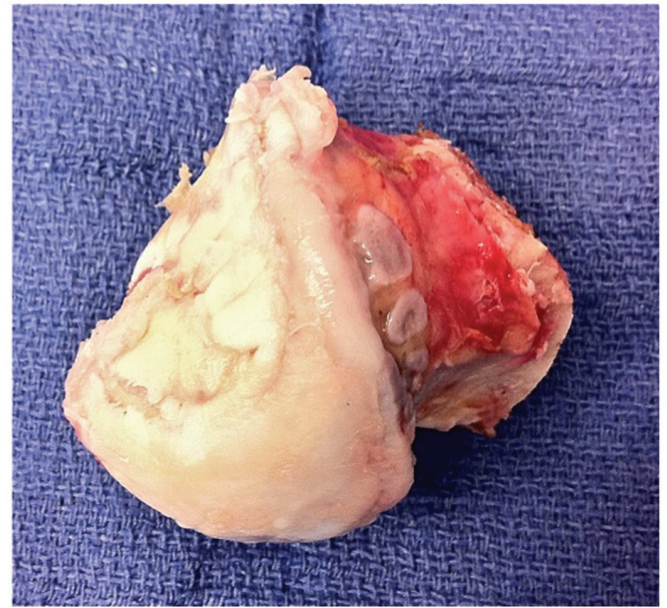

Figure 5. Right femoral head with undamaged cartilage on inferior aspect. ed by avascular necrosis with an overall shape potentially suitable for bulk osteochondral reconstruction of the lateral femoral condyle. A provisional surgical plan was made to proceed with right total hip arthroplasty and simultaneous osteochondral reconstruction of the right lateral femoral condyle, if the resected femoral head was judged to be suitable for auto-grafting. This procedure was preceded by left total hip arthroplasty by a direct anterior inter-muscular approach; however, the left femoral head was considered unsuitable for use in osteochondral auto-grafting on the basis of global articular surface damage.

Two weeks after left total hip replacement the patient

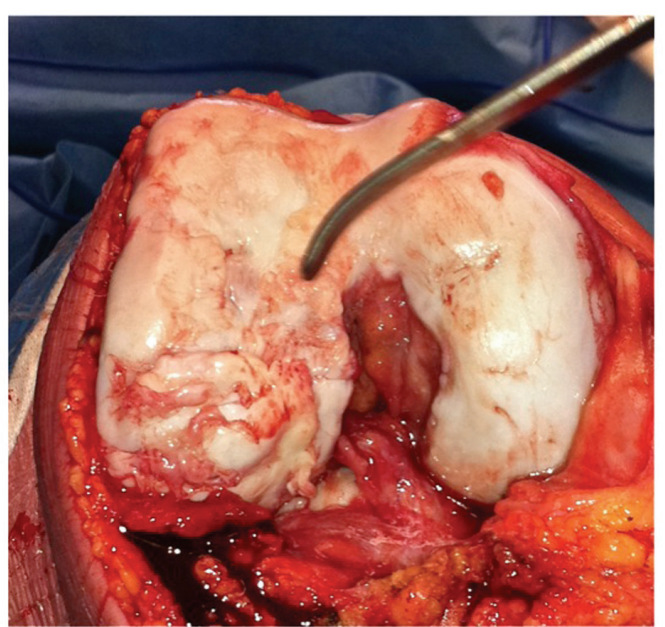

Figure 6. Exposure of lateral femoral condyle. 


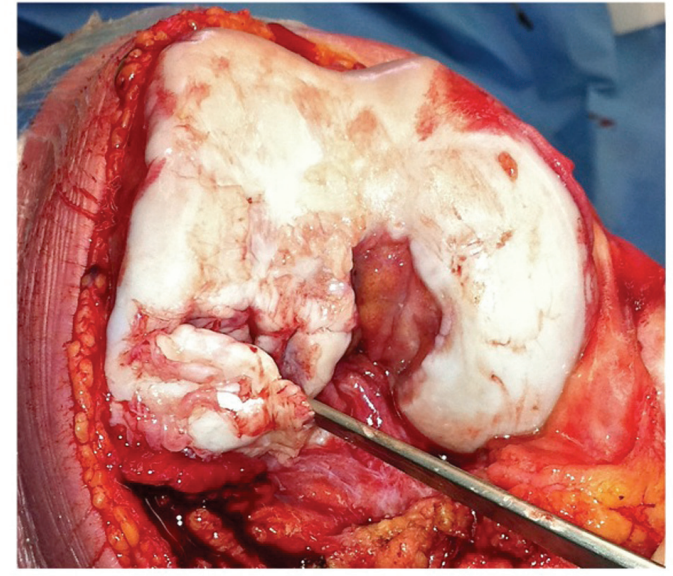

Figure 7. Resection of avascular bone from lateral femoral condyle.

underwent right total hip arthroplasty via a direct anterior intermuscular approach. Particular care was made during dislocation of the joint and resection of the femoral neck to avoid damage to the posterior and inferior portion of the femoral head. The femoral head was found to have undamaged cartilage surfaces in the inferior aspect with good quality sub-articular bone (Fig. 5), and judged suitable to proceed with the proposed knee joint reconstruction procedure. A second surgical team prepared the graft while the total hip arthroplasty procedure was completed.

Exposure of the right knee was achieved through a midline incision and a medial para-patellar arthrotomy (Fig. 6). The involved areas of the lateral femoral condyle were resected, resulting in a trapezoidal defect of $15 \mathrm{~mm}^{2}$; native bone was retained where viable, creating a vascular bed of cancellous bone (Fig. 7). The trapezoidal shape of the re-

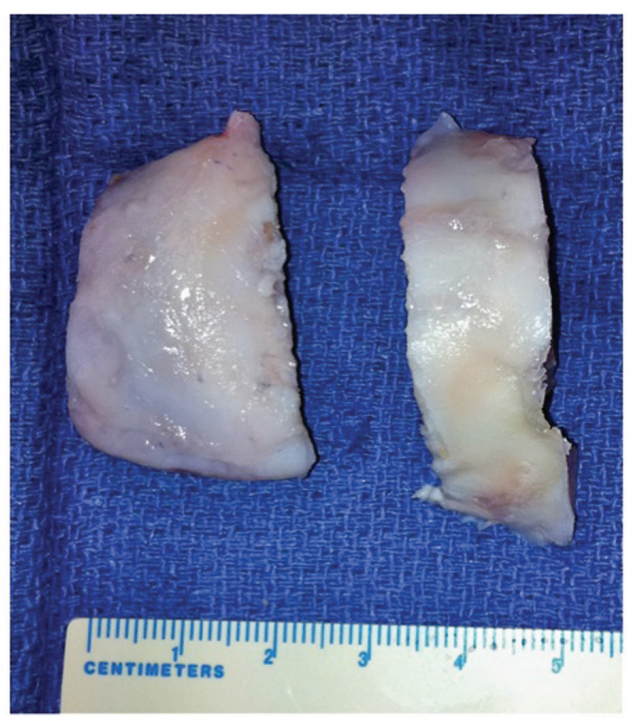

Figure 8. Shaped autograft sections from femoral head.

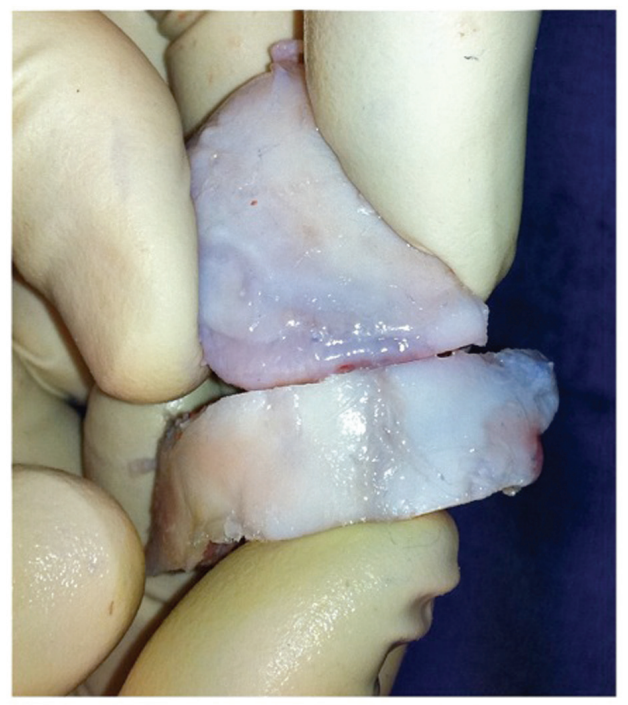

Figure 9. Reproduction of the desired elliptical contour of the lateral condyle.

section facilitated primary stability of the bulk auto-graft; the graft was prepared to best match the shape of the osteochondral defect. The intact articular surface of the femoral head was unfortunately discontinuous, and the autograft was therefore harvested in two distinct sections (Fig. 8). To reproduce the relatively elliptical contour of the distal femoral articular surface compared to the spherical femoral head, an incomplete osteotomy of the autograft fragments was conducted to the level of subchondral bone (Fig. 9). The thickness of the auto-graft was maintained at a minimum of $10 \mathrm{~mm}$ throughout. After graft impaction and final seating, internal fixation was performed using multiple headless compression screws (Fig. 10). Very satisfactory patello-femoral tracking and coronal plane stability of the knee was confirmed after completion of the procedure, prior to closure.

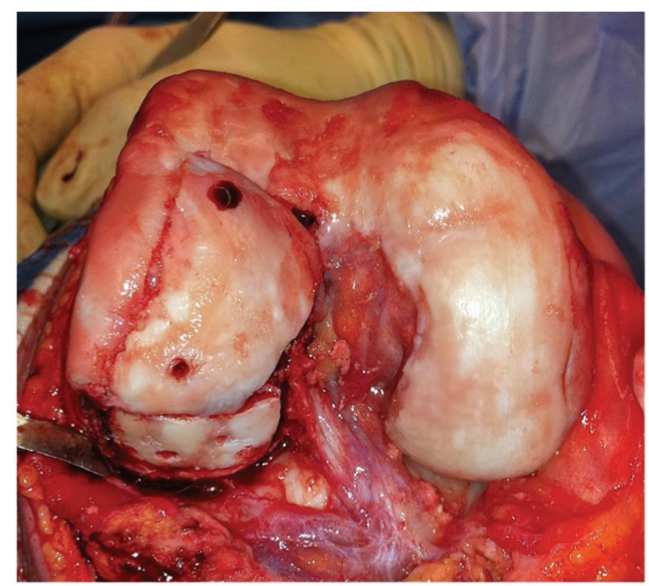

Figure 10. Fixation of the autograft into position using headless compression screws. 


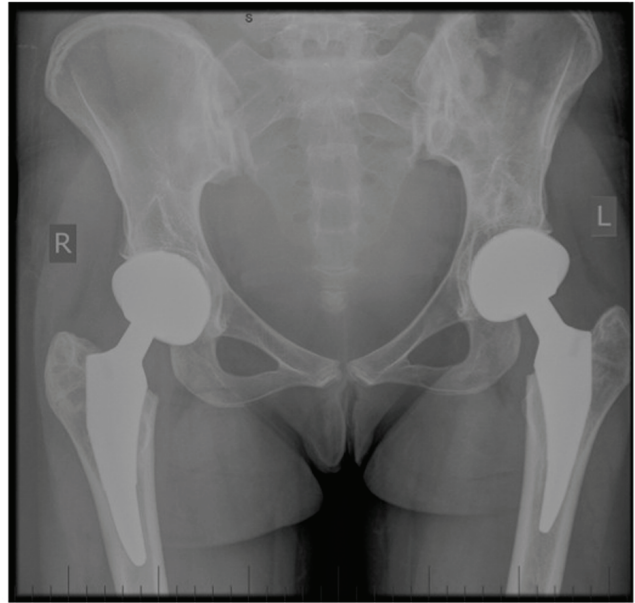

Figure 11. Postoperative AP pelvis x-ray.

Postoperative rehabilitation included immediate full weight-bearing on the left hip arthroplasty. The right knee and hip underwent a graduated program of protected activity with touch weight bearing for a period of six weeks, progressing to partial weight bearing for a further six weeks. Early knee range of motion exercises were permitted as tolerated without restriction. Seven weeks after the procedure both hips were pain free; the right knee demonstrated a $15^{\circ}$ fixed flexion deformity, and flexion had returned to greater than $130^{\circ}$. At six months post surgery the knee exhibited full extension and flexion to $150^{\circ}$, with only a mild ache on prolonged walking.

At 12 months post-operative both hips remained pain free and radiographs demonstrated the hip implants were stable and well osseo-integrated (Fig. 11). The Modified Harris Hip Score (mHHS) results improved from 37.4/100 (preoperatively) to $100 / 100$ (post-operatively), and the NonArthritic Hip Score (NAHS) improved from 40/84 to 72/84. The right knee was causing only very minor and occasional discomfort about the medial aspect. There was no effusion and the range of motion was almost equal to the contralateral side, with full extension and flexion to $160^{\circ}$ (Fig. 12). The Knee Outcome Score (KOS) 12 months after the procedure was $113 / 125$. On further physical examination the knee remained stable to varus/valgus stress, patellar tracking was unremarkable, and there was no demonstrable ligamentous instability. Radiographs of the right knee confirmed the bulk osteochondral autograft had united completely and remained viable, with no evidence of necrosis, loss of reduction, or change in position of the hardware (Fig. 13). The knee joint surfaces appeared smooth, with a contour very closely approximating normal.

\section{Discussion}

This young woman initially presented with a polyarticular osteonecrosis as a complication of the chemotherapy used in the management of her acute lymphocytic leukaemia (ALL). During the course of her management, she developed marked symptoms involving both hips and one knee after the articular surfaces of both femoral heads and the right knee lateral femoral condyle all collapsed, resulting in significant pain and disability. For this particular case a novel technique

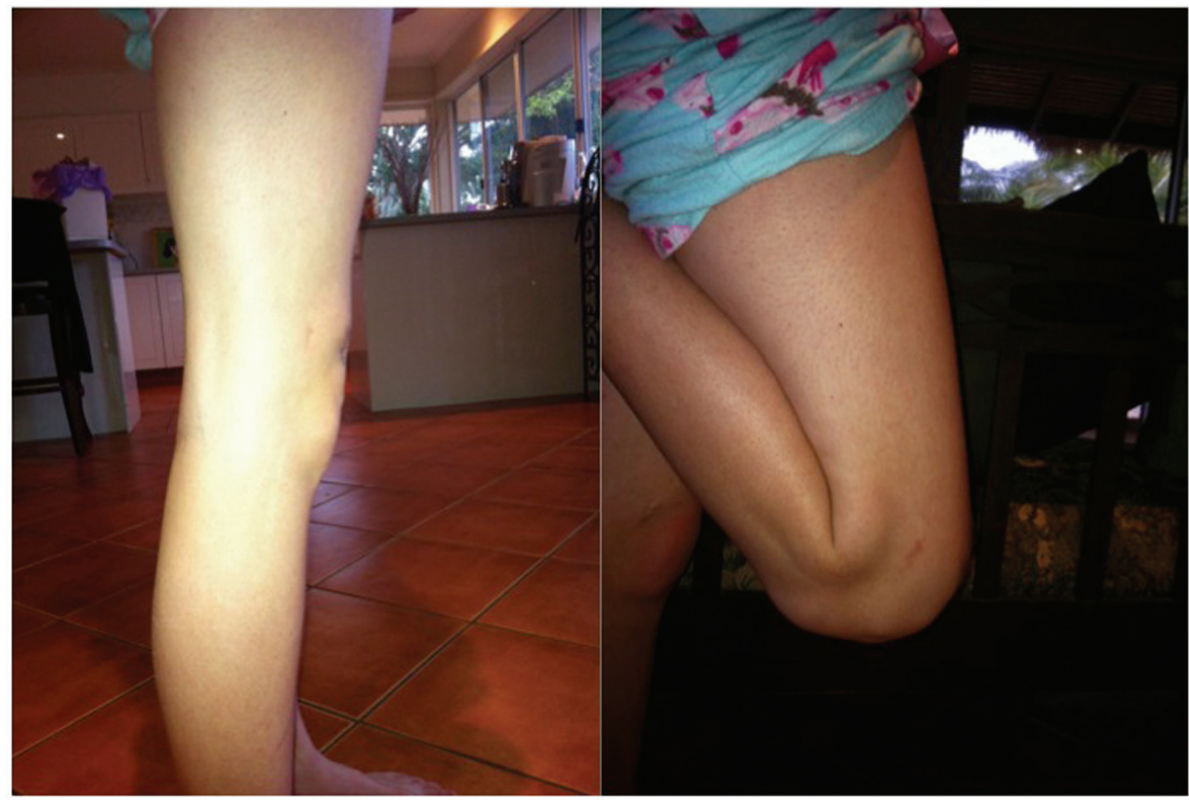

Figure 12. Full extension and flexion of right knee. 


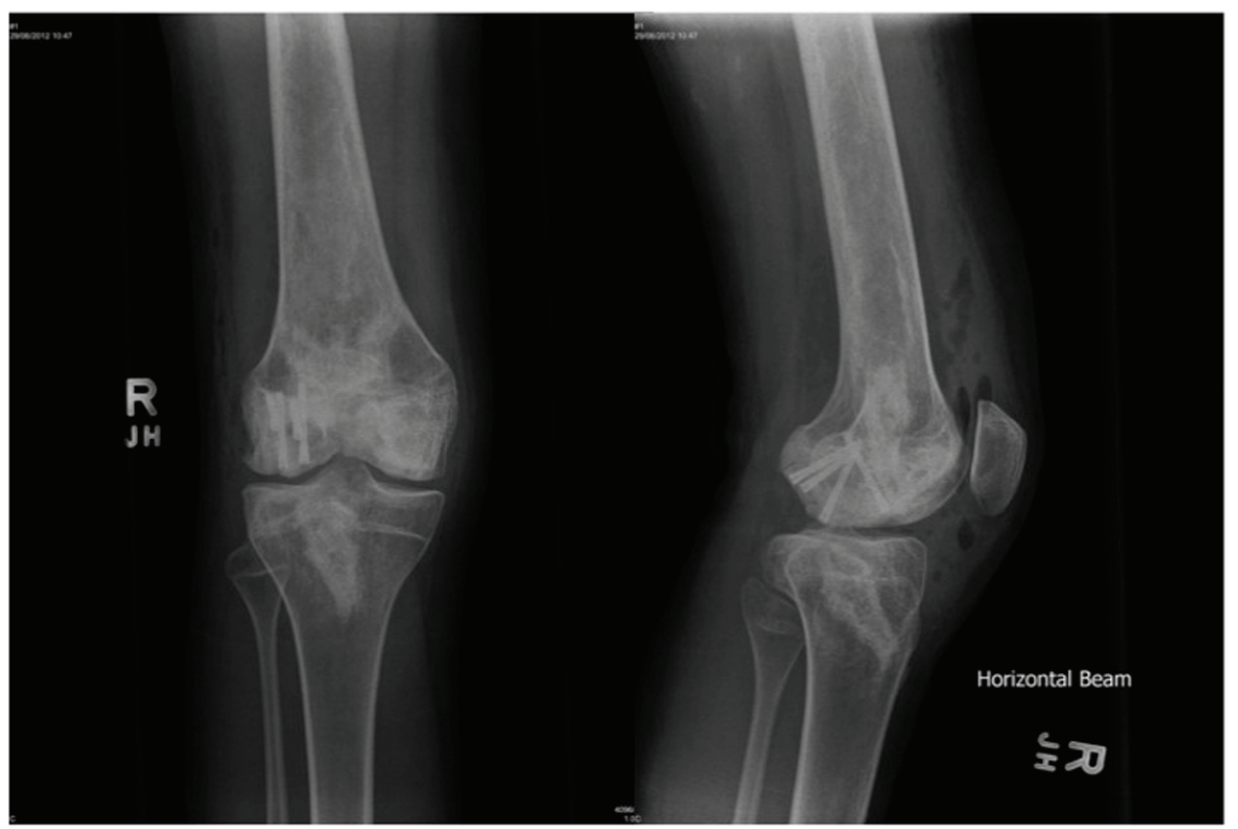

Figure 13. Postoperative AP and Lateral right knee x-rays.

was employed, cognizant of the various limitations imposed by the number of joints involved, the nature and size of the lesions, the patients age, and the difficulties inherent in procuring a suitable fresh osteochondral allograft. We chose to reconstruct the articular surface of the knee using a fresh osteochondral autograft harvested from the ipsilateral femoral head during a simultaneous total hip arthroplasty, a technique we believe to be novel and unique.

After failing non-operative measures, management options would generally include arthroplasty, arthrodesis, and osteochondral grafting, either allograft or autograft. Multiple factors would influence this decision, including which joint is involved, the size of the lesion, bilaterality, the age of the patient, and the number of joints involved. Further factors may include specific considerations such as patient expectations, anticipated demands, and the surgeons training and experience.

In such a young patient the treatment options are further limited, and definitive reconstruction by joint arthroplasty may be considered less palatable. Total hip replacement is generally regarded as the most consistent and predictable arthroplasty, with the greatest potential for adequate longevity to warrant the considerable risks associated. In this patient the polyarticular nature, female gender, and bilaterality all precluded hip arthrodesis as an option, and we elected to manage her hip pathology with bilateral uncemented total hip replacements. Bulk osteochondral grafting of the lateral femoral condylar lesion was our preferred option.

In this case, fresh osteochondral auto-grafting enabled rapid osseous integration and articular surface reconstruction using living host cartilage, resulting in an excellent clinical outcome. Although uncommon, concurrent avascular necrosis of multiple large joints is occasionally encountered, and management options are particularly limited in young patients. We recommend this innovative method be considered in those patients who satisfy the criteria for bulk osteochondral grafting procedures where total hip replacement is also required.

While this procedure would be suitable to perform using any preferred surgical approach, we believe the use of an anterior inter-muscular approach has particular advantages in complex cases such as this to facilitate more rapid rehabilitation. In this situation, after 2 weeks our patient had recovered sufficiently from her left hip replacement to proceed with her combined right hip and knee procedure and safely mobilise touch weight bearing in the immediate postoperative period. Performing the right hip procedure by the anterior approach further assisted in her rehabilitation following her combined procedures.

Corticosteroids are used routinely as part of the chemotherapy treatment regimen for many neoplastic conditions, including ALL. Unfortunately, one of the recognised and established complications of this treatment is osteonecrosis, which can be particularly debilitating when it involves weight-bearing joints. In a prospective MRI study, Ojala et al [1] reported development of osteonecrosis in 32\% (9 of 28) of patients during treatment for ALL, although only four of those nine were symptomatic. The management of this condition poses particular challenges given the typical juvenile onset of ALL. Joint preserving strategies dominate management efforts, avoiding joint replacement whenever possible in patients so young [2]. 
Werger et al [2] reviewed eight patients who developed osteonecrosis during treatment for ALL and Non-Hodgkin's lymphoma, and discussed the various management strategies available. Potential options for the knee included core decompression, cancellous bone transplantation (autologous or allogeneic) combined with collagen pads, bone substitutes, periosteal flaps, and osteochondral autografts harvested from the ipsilateral or contralateral knee. Two patients in this study also required concomitant hip replacements; however, use of the femoral head for osteochondral grafting was not employed. Fujita et al [3] presented a case series of three young patients $(18,24$ and 25 years) who all developed osteonecrosis of the knee secondary to steroid use for chronic renal conditions. All three underwent autologous osteochondral grafting to the affected weight-bearing surface, and two also underwent micro-fracture for treatment of chondral defects in non-weight-bearing areas. They reported satisfactory clinical outcomes with symptomatic improvement, and at two years post-operative both MRI and arthroscopy demonstrated viability and incorporation of the grafts.

Gortz et al [4] have reported the largest case series of young patients with osteonecrosis of the knee related to corticosteroid use, managed by the use of fresh osteochondral (shell or plug) allografts to the affected femoral condyles. Their study cohort included 22 patients (28 involved knees) with an average age of 24.3 years (16 - 44). They reported promising results, with 18 of 19 surviving participants having a significant reduction in the severity of their symptoms; only two of the 14 osteochondral allografts with long-term follow up demonstrated radiographic collapse. Three of their cases were later revised, with only one requiring a TKA at 78 months secondary to pain.

Multiple small case series [5-9] have described the success of osteochondral autografts for treatment of osteochondral defects in the knee, including cases of spontaneous osteonecrosis of the knee (SONK), secondary osteonecrosis, or osteochondritis dissecans. As for other small osteochondral defects, osteochondral autograft plugs are harvested from the periphery of either the ipsilateral or contralateral knees. The recent literature review by Lieberman et al [10] critically examined the evidence for various surgical procedures in treatment of osteonecrosis of the knee. The evidence is unfortunately limited, principally due to the paucity of available cases and the lack of randomised controlled studies. Core decompression was a successful technique, with better results achieved prior to collapse and with smaller osteochondral defects. Osteochondral grafting techniques employed in knees were successful both pre-collapse ( $0 \%$ failure) and post-collapse ( $8.2 \%$ failure). The success of this procedure in knees has not been replicated in hips, which typically continue to collapse and usually later require arthroplasty.

Having conducted a thorough review of the available literature, the technique we describe here appears to be unique. Harvesting the remaining intact articular surfaces of the femoral head during a simultaneous total hip arthroplasty for use as an immediate osteochondral autograft transfer to the knee has not been previously described. Although distinctly uncommon, this clinical scenario is not rare and we believe other patients may obtain genuine benefit from this novel treatment strategy.

\section{Conclusion}

We have described here a unique case of successful management of lateral femoral condyle osteonecrosis by use of fresh femoral head autograft obtained during simultaneous total hip arthroplasty. To the best of our knowledge this paper reports on the first, and to date only, use of this innovative technique. The use of a fresh osteochondral autograft has a number of advantages; we recommend this technique be considered in those unusual situations where total hip arthroplasty is contemplated in patients also requiring bulk osteochondral grafting procedures to the knee or other large joints.

\section{Disclosure}

The author/s report that there is no conflict of interest regarding the material discussed in this manuscript.

Ethical approval for this paper was sought and approved by UnitingCare Health - Human Research Ethics Committee on $30 / 07 / 2013$.

This procedure was conducted at St Andrew's War Memorial Hospital, Brisbane, Australia on the 27th June 2012.

\section{References}

1. Ojala AE, Lanning FP, Paakko E, Lanning BM. Osteonecrosis in children treated for acute lymphoblastic leukemia: a magnetic resonance imaging study after treatment. Med Pediatr Oncol. 1997;29(4):260-265.

2. Werner A, Jager M, Schmitz H, Krauspe R. Joint preserving surgery for osteonecrosis and osteochondral defects after chemotherapy in childhood. Klin Padiatr. 2003;215(6):332-337.

3. Fujita N, Matsumoto T, Kubo S, Matsushita T, Ishida $\mathrm{K}$, Hoshino Y, Nishimoto K, et al. Autogenous osteochondral graft transplantation for steroid-induced osteonecrosis of the femoral condyle: A report of three young patients. Sports Med Arthrosc Rehabil Ther Technol. 2012;4(1):13

4. Gortz S, De Young AJ, Bugbee WD. Fresh osteochondral allografting for steroid-associated osteonecrosis of the femoral condyles. Clin Orthop Relat Res. 2010;468(5):1269-1278.

5. Imade S, Kumahashi N, Kuwata S, Iwasa J, Uchio Y. Effectiveness and limitations of autologous osteochon- 
dral grafting for the treatment of articular cartilage defects in the knee. Knee Surg Sports Traumatol Arthrosc. 2012;20(1):160-165.

6. Kotani A, Ishii Y, Sasaki S. Autogenous osteochondral grafts for osteonecrosis of the femoral condyle. J Orthop Surg (Hong Kong). 2003;11(2):117-122.

7. Matsusue Y, Kubo M, Nakagawa Y. Autogenous Bonecartilage Transplantation. Techniques in Knee Surgery. 2010;9:85-94. doi:10.1097/BTK.0b013e3181e0a139.

8. Okamoto Y, Nakagawa Y, Maekawa M, Kobayashi M, Nakamura T. Osteochondral grafting for treatment of a massive chondral defect in the knee of a young adult with anterior cruciate ligament deficit. Arthroscopy. 2007;23(9):1024 e1021-1024.

9. Tanaka Y, Mima H, Yonetani Y, Shiozaki Y, Nakamura $\mathrm{N}$, Horibe S. Histological evaluation of spontaneous osteonecrosis of the medial femoral condyle and shortterm clinical results of osteochondral autografting: a case series. Knee. 2009;16(2):130-135.

10. Lieberman JR, Varthi AG, Polkowski GG, 2nd. Osteonecrosis of the Knee - Which Joint Preservation Procedures Work? J Arthroplasty. 2013. 